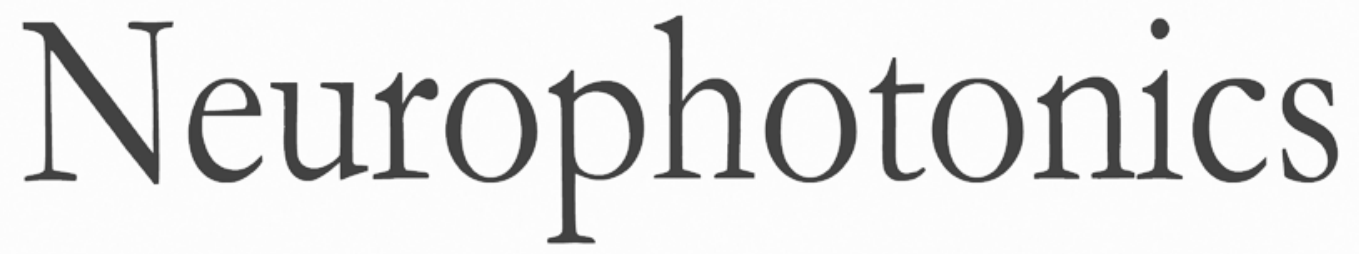

\title{
Review: How do spontaneous and sensory-evoked activities interact?
}

\author{
Isabelle Ferezou \\ Thomas Deneux
}




\title{
Review: How do spontaneous and sensory-evoked activities interact?
}

\author{
Isabelle Ferezou and Thomas Deneux ${ }^{\star}$ \\ Unité de Neurosciences, Information et Complexité, Centre National de la Recherche Scientifique, Gif-sur-Yvette, France
}

\begin{abstract}
Twenty years ago, the seminal work of Grinvald et al. revolutionized the view cast on spontaneous cortical activity by showing how, instead of being a mere measure of noise, it profoundly impacts cortical responses to a sensory input and therefore could play a role in sensory processing. This paved the way for a number of studies on the interactions between spontaneous and sensory-evoked activities. Spontaneous activity has subsequently been found to be highly structured and to participate in high cognitive functions, such as influencing conscious perception in humans. However, its functional role remains poorly understood, and only a few speculations exist, from the maintenance of the cortical network to the internal representation of an a priori knowledge of the environment. Furthermore, elucidation of this functional role could stem from studying the opposite relationship between spontaneous and sensory-evoked activities, namely, how a sensory input influences subsequent internal activities. Indeed, this question has remained largely unexplored, but a recent study by the Grinvald laboratory shows that a brief sensory input largely dampens spontaneous rhythms, suggesting a more sophisticated view where some spontaneous rhythms might relate to sensory processing and some others not. @ The Authors. Published by SPIE under a Creative Commons Attribution 3.0 Unported License. Distribution or reproduction of this work in whole or in part requires full attribution of the original publication, including its DOI. [DOI: 10.1117/1.NPh.4.3.031221]
\end{abstract}

Keywords: spontaneous activity; sensory processing; cerebral cortex; brain states.

Paper 17010SSVR received Jan. 17, 2017; accepted for publication May 16, 2017; published online Jun. 13, 2017.

\section{Spontaneous Activity Influences Evoked Responses}

Investigating the features detected by individual neurons or by neuronal assemblies has been one of the most successful approaches to understanding brain organization and function. This approach requires measuring the neuronal responses to a set of different sensory inputs; as a consequence, the variability of these responses between different presentations of the same stimulus has long been considered a disturbance that needed to be overcome by trigger-averaging over a number of presentations. This unfortunately led to the disregard of this variability in evoked responses-as well as the large activity fluctuations observed in the absence of stimulation-and rather consider them as noise.

Twenty years ago, however, Arieli et al. ${ }^{1}$ focused their interest on these response variabilities and spontaneous ongoing activity, using single-neuron electrical recordings coupled with voltage-sensitive dyes (VSDs) to measure coherent activities in the visual cortex of anesthetized cats. They observed, in particular, that ongoing fluctuations and response variabilities had amplitudes as large as the evoked responses, were highly correlated between neurons as far as 6-mm apart, and showed structure in both space and time. This led them to emphasize the importance of studying these activities as they speculated that the "ongoing electrical activity and its specific interactions with the activity evoked by the stimulus may be one neuronal expression of context." This speculation was greatly confirmed by their next report ${ }^{2}$ where they showed that the variability in response patterns evoked by individual stimulus presentations

*Address all correspondence to: Thomas Deneux, E-mail: thomas.deneux@ unic.cnrs-gif.fr could be well accounted for by the ongoing patterns that immediately preceded the stimulation [Fig. 1(a)]. This evidence for integration of a deterministic response to the sensory input with the ongoing network dynamics reinforced their argument that ongoing activity "may provide the neural substrate for the dependence of sensory information processing on context and on behavioral and conscious states."

They succeeded in triggering a new consideration for ongoing dynamics, and a large number of studies that followed investigated how spontaneous activity patterns influence the responses to specific stimulations. ${ }^{4-23}$ It is noteworthy that the positive correlation that they reported between ongoing activity and sensory-evoked responses was soon contradicted and that a wider range of interactions was subsequently reported. Indeed, Petersen et al. ${ }^{7}$ observed, in the barrel cortex of anesthetized rats, that sensory-evoked responses were much stronger when ongoing activity was low compared to when it was high. More precisely, the ongoing activity in this preparation showed characteristic up and down states, ${ }^{24}$ where the whole network activity in a local neighborhood alternates between periods of tonic activity (up), possibly propagating as waves, and silence (down). During such synchronized cortical states, which can also be observed during quiet wakefulness, ${ }^{6,7,14}$ responses evoked by tactile or tone stimuli are typically of large amplitude and are inversely correlated to the prestimulus membrane potential. ${ }^{7,15,23}$ The sensory-evoked cortical responses are further suppressed when the cortex switches from slow wave activity to a more desynchronized state, typical of active wakefulness. ${ }^{6,14}$ Such suppression of evoked cortical activity occurring during behaviorally active states has been reported both in the primary somatosensory (see also Refs. 25 and 26) and primary auditory cortex. ${ }^{27-29}$ However, several recent studies indicate that the interaction 


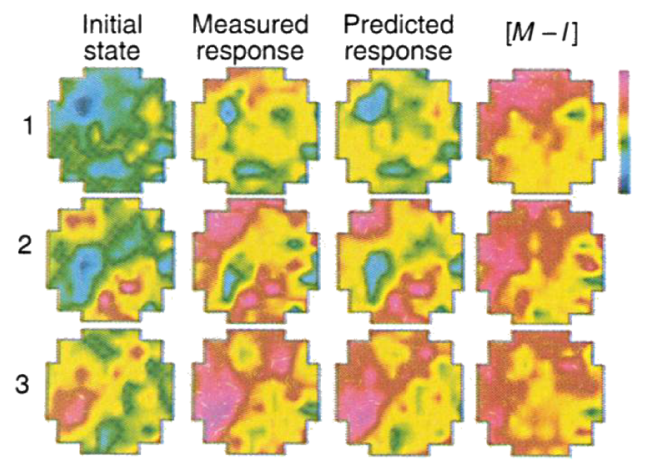

(a)

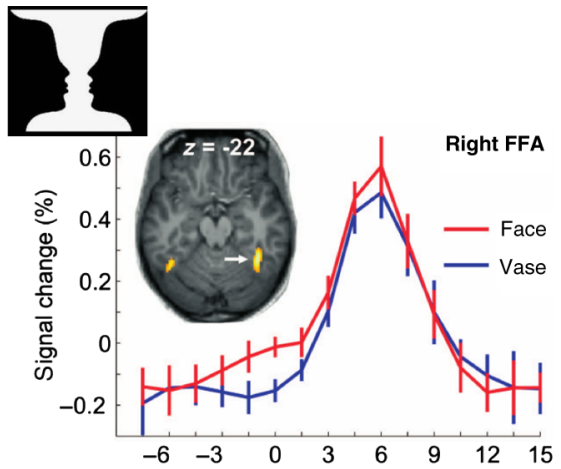

(b)

Fig. 1 Spontaneous activity influences evoked responses: (a) three consecutive single-trial responses (rows) to the same visual stimulus, showing the initial state, the measured response $28 \mathrm{~ms}$ later, and the predicted response obtained by simple summation of initial state and the average response to all trials. Subtracting the initial state from the measured response yielded the net pattern $(M-l)$. Reproduced from Ref. 2 with permission. (b) Peristimulus fMRI signal time courses from right FFA in response to an ambiguous face-vase image (inset), averaged across subjects, after sorting trials according to whether they reported a face or vase perception: face perception was associated with a higher prestimulus activation level. Adapted from Ref. 3 with permission.

between behavioral activity, cortical state, and sensory-evoked responses is opposite in the primary visual cortex..$^{4,22,30-35}$ The interplay between ongoing cortical dynamics and sensory inputs, therefore, does not seem to follow common rules across sensory modalities. Furthermore, by recording the membrane potential of mice engaged in a tactile detection task, a recent study from Petersen $1 \mathrm{ab}^{36}$ revealed that, although the ongoing cortical state impacts the evoked sensory response, it has no effect on the performance of the animal.

In the human neuroscience community, the study of ongoing dynamics has met a great interest ${ }^{3,37-42}$ (see Ref. 38 for a review). This stems from the interest for high cognitive functions in humans, such as imagination or consciousness, of which ongoing activity could be a hallmark [see also later our mention to the "default mode network (DMN)"]. The influence of ongoing cortical dynamics on the processing of sensory inputs was also established. As an example, Hesselmann et al. ${ }^{3}$ found using fMRI that the perception of a flashed ambiguous face-vase stimulus depended of prestimulation activity level in the fusiform face area (FFA) and an extrastriate visual region specialized for face processing as well as in some other brain areas [Fig. 1(b)]. It thus appears that even conscious perception cannot be considered independently of the "initial state of the system," to take the author's words, and that the measured spontaneous activity signals, even though their functional meaning remain unfathomed, constitute at least a fingerprint of this initial state.

\section{Structure of Spontaneous Activity Reflects Functional Organization and is Influenced by Experience}

The Grinvald laboratory made other keystone contributions to the study of spontaneous activity by taking advantage of the exquisite topographical organization of the cat visual cortex, on the one hand, and of VSDs on the other hand, to capture this organization. Functional structures usually revealed by sensory stimulation were also found in the spontaneous dynamics: at the level of a single-neuron functional connectivity, ${ }^{43}$ where population activity maps trigger-averaged on a single-neuron spikes appeared to be near-identical in the resting or stimulation conditions; and at the level of the population representations, ${ }^{44}$ where spontaneous activity patterns were observed, which highly resembled evoked orientation maps [Fig. 2(a)].

That the spontaneous activity reflects the functional organization of the cortical network on which it is riding is not a surprise and has been confirmed at the scale of the whole dorsal surface of cortical hemispheres in mice by VSD imaging. ${ }^{47}$

However, the spectacular aspect of spontaneously emerging orientation maps raised a new question: can the spontaneous cortical states play an active role in sensory processing, as the authors suggested that they might "reflect expectations about the sensory input?"

An additional relationship between sensory-evoked and spontaneous activities lies in the plasticity of the latter, in the sense that sensory-evoked activity can reshape the structure of subsequent spontaneous patterns through learning. This was shown in particular by Dan group ${ }^{45,48}$ who, after training rats with visual stimuli to evoke wave patterns in their primary visual cortex, observed recalls of these specific patterns in the spontaneous activity during the resting period that followed [Fig. 2(b)]. Such recalls or replays are in fact a phenomenon that is well-known and abundantly studied, in particular, in hippocampal structures. ${ }^{49-54}$

The similarities in structure between spontaneous and sensory-evoked activities might, therefore, be learned through experience rather than innately. In this light, Berkes et al. ${ }^{46}$ emphasized changes that occur during development, whereby an initial mismatch between the statistics of spontaneous and sensory-evoked (using natural visual stimuli) activities in young ferrets disappears in adult animals [Fig. 2(c)]. There again it is suggested that spontaneous activity reflects prior expectations of "an internal model (of the natural environment) that is adapted gradually during development."

Moreover, spontaneous activity is known to play an active role during development, in particular, in the early stages of development where propagating waves of activity are known to shape and consolidate the developing networks (see reviews in Refs. 29 and 55, as well as the review from Luhmann ${ }^{56}$ in this issue of Neurophotonics). This is a whole field of investigation 


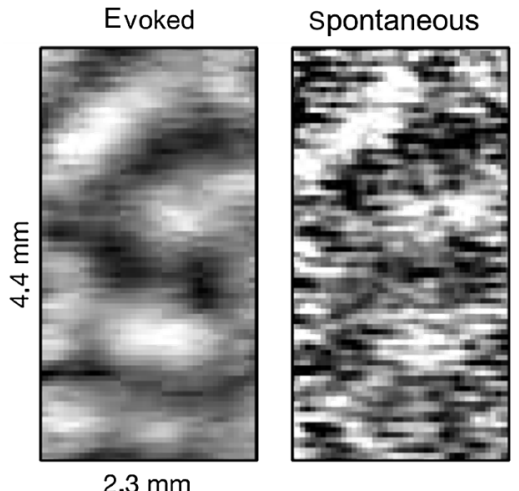

(a)

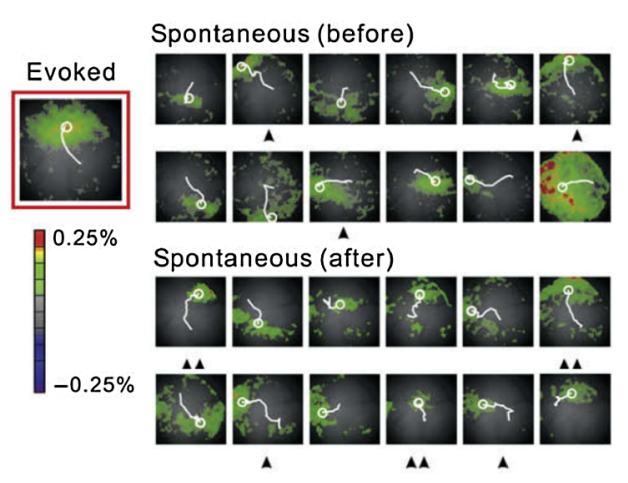

(b)

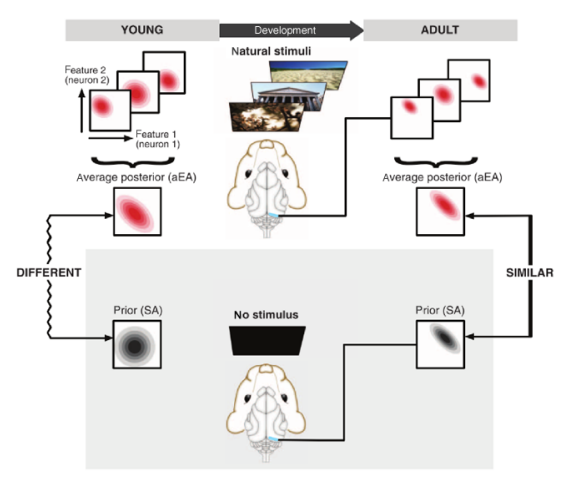

(c)

Fig. 2 The structure of spontaneous activity reflects functional organization and is influenced by experience: (a) an activation pattern obtained from a single frame from spontaneous activity VSD recording (right) matches the orientation map obtained by averaging responses to full-field gratings of vertical orientation (left), in the visual cortex of an anesthetized cat. Adapted from Ref. 43 with permission. (b) Spontaneous waves recorded using VSD in an anesthetized rat barrel cortex, immediately before and after training with a flashing sequence that evoked the wave template represented on the left. Waves are represented by their first frame and the trajectory of their center over $\sim 160 \mathrm{~ms}$. Spontaneous waves well matched to the template are indicated by a single arrowhead [correlation coefficient $(C C)>0.6$ ] or by double arrowheads $(C C>0.7)$ and are more frequent after training. Adapted from Ref. 45 with permission. (c) Multiunit activity recorded in V1 of awake, freely viewing ferrets either receiving no stimulus (bottom) or viewing natural (top) or artificial stimuli (not shown in this adapted figure) is used to construct neural activity distributions in young and adult animals. Distributions of evoked activities averaged over different stimuli are compared with the distribution of spontaneous activities, assumed to represent the prior expectations about visual features. The internal model of young animals (left) is expected to show little adaptation to the natural environment and thus show a mismatch between spontaneous and evoked distributions. On the contrary, adult animals (right) are expected to be adapted to natural scenes and thus to exhibit a high degree of similarity. Adapted from Ref. 46 with permission.

in itself, and it is not obvious how these spontaneous activities during early development relate to those observed in adults.

Plasticity in the spontaneous activity structure has also been shown in humans. Lewis et al. ${ }^{57}$ found that a stimulated part of the visual cortex modified its resting-state connectivity after training as compared to the untrained part. A few studies have investigated changes in the resting-state network induced by preceding task periods involving memorization or emotional content (see Ref. 38 for review).

Whereas we started this retrospective review with the influence of spontaneous activity on sensory-evoked responses, ${ }^{2}$ we have now discussed influences in the opposite direction through learning. However, another important question has been raised: does the spontaneous activity really embed a representation of "expectations" in such a way that it plays an active role during sensory processing? To address this question, a new level of interaction is envisioned: how are spontaneous dynamics affected by a sensory inflow?

\section{Sensory Input Switches the Brain Internal Dynamics}

\subsection{Brain Dynamics during a Sensory Input}

"Stimulus onset quenches neural variability: a widespread cortical phenomenon," 58 under this title, a number of well-known neuroscientists gathered 14 different electrophysiology datasets recorded in cats and monkeys, which all showed that intertrial variability decreased in sensory-evoked responses as compared to the preceding period of spontaneous activity [Fig. 3(a)], fluctuations present in the spontaneous activity [Fig. 3(a), top] systematically decreased in amplitude during stimulation, even in instances where this stimulation was not eliciting an "average response" [Fig. 3(a), middle] (Note that, even though it is artificial to split the signals after stimulus onset between an "average response" and "remaining fluctuations," we chose to call these fluctuations "internal" or "internally generated" activity, as obviously it cannot be called a "spontaneous activity"). This phenomenon actually was already known from intracellular studies ${ }^{61}$ that showed how a sensory input resulted in very reproducible driving of a neuron membrane potential as compared to the spontaneous fluctuations and identified shunting inhibition as a mechanism for the rescaling of the cell excitability.

Even though it appears intuitive that a sensory drive might "clamp" the firing dynamics to fixed patterns and therefore reduce the variability due to random fluctuations generated by the network itself, neural simulations revealed interesting properties of this general effect. For example, the work of Abbott group $^{62,63}$ showed not only that variability reduction was an intrinsic property of interconnected networks shifting from chaotic to driven dynamics when exposed to an input but also that complex nonlinear interactions occurred between intrinsic and sensory-driven dynamics. These included the preference for some input frequency without any resonance effect, the drive at harmonic frequencies initially not present in the input, and the curving of the spatial patterns of the input toward those of the intrinsic dynamics. Some of these effects were later confirmed experimentally. ${ }^{64}$ On the other hand, other computational neuroscientists have advocated that the reduction of variability corresponds to a very peculiar structural property of the brain network, such that its activity spans a highly multidimensional space "at the edge" of multiple bifurcations, leading to multiple 

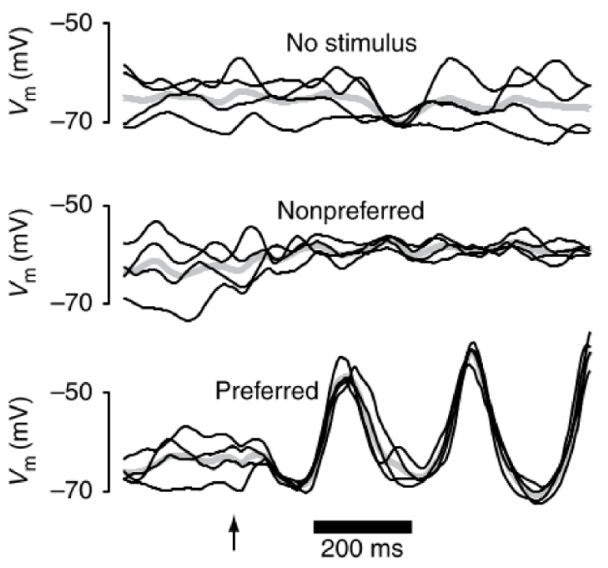

(a)

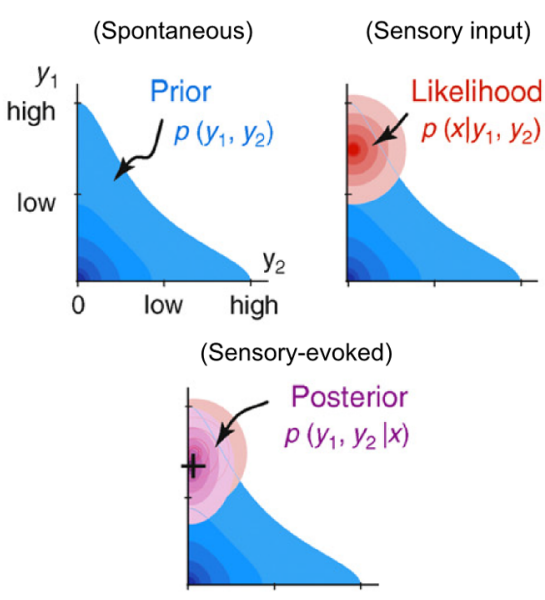

(b)
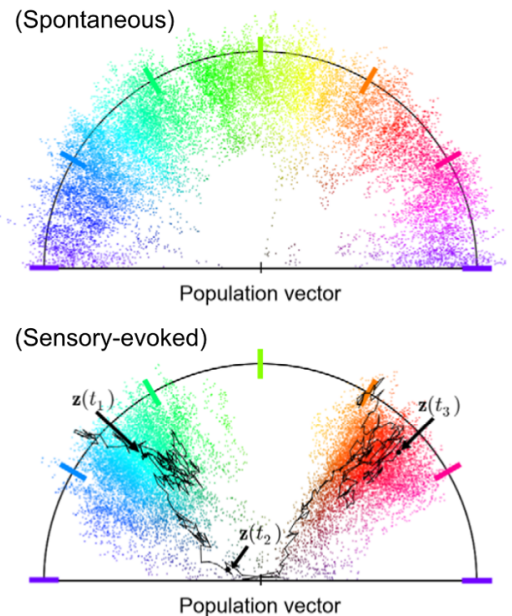

(c)

Fig. 3 Brain dynamics during a sensory input: (a) example intracellular recordings from a neuron in cat V1. Intertrial variability decreases in stimulated recordings for both nonpreferred (middle) and preferred (bottom) orientations and frequencies of the sine-wave gratings as compared to unstimulated recordings (top). Reproduced from Ref. 58 with permission. (b) Schematic illustration of probabilistic inference in sensory processing. The information brought by a priori knowledge about the environment and by the sensory input are represented, respectively, as a prior and likelihood probability distributions (top). These two types of information are optimally combined by Bayes' theorem, forming a posterior probability distribution that displays less uncertainty (is narrower) than the two previous distributions (bottom). Adapted from Ref. 59 with permission. (c) Simulations of a small population of neurons inferring the orientation of a grating by implementing a sampling of the above-mentioned distributions. Individual neurons in these simulations are tuned to different orientations and are preferentially connected to neurons with similar preferences. Individual points in the graphs represent the activity of the full population at different time points, their color and angular position encoding the orientation of the most active neurons, and their radius of the population coherence. In the spontaneous activity, the population activity wanders throughout all orientations, representing the prior distribution (top), whereas, in presence of an ambiguous input, its wandering is restricted to the possible orientations as constrained by the input, representing the posterior distribution (bottom). Adapted from Ref. 60 with permission.

"ghost attractors." ${ }^{, 65,66}$ In the absence of a sensory input, the spontaneous activity can visit a large repertoire of states; however, even a weak external input can lead it to fall into one of the attractors, which decreases variability.

In addition to this modeling effort, a functional role in sensory processing was proposed: ${ }^{59,60,67}$ the spontaneous activity, by sampling a large ensemble of states, maintains an internal representation of all possible external environments and thereby implements an "expectation" or, in Bayesian terms, a "prior." Once combined with the information brought by a sensory input about the actual state of the external world, this prior is reshaped into a "posterior," which by essence embeds less uncertainty and therefore restricts the number of sampled states [Figs. 3(b) and 3(c)].

According to this theory, the spontaneous activity is viewed as playing an active role in sensory processing, the network being permanently in an attempt to make inferences about the environment, even under resting condition or sleep where it explores all possibilities learned from accumulated experience. Because sensory-evoked activity is then viewed as a combination of the information entailed in the internal dynamics, on the one hand, and in the sensory input, on the other hand, it gives a functional significance to the above-mentioned patterns of integration between spontaneous fluctuations and sensoryevoked activities, as well as the reshaping of spontaneous activity by experience. This view, however, appears quite restrictive in regard to some stereotypic and widespread spontaneous rhythms, which are unlikely to achieve a "sampling of internal representations," such as the up and down fluctuations. ${ }^{24}$

\subsection{Brain Dynamics after a Sensory Input}

To further investigate the interactions between spontaneous and evoked activities, Deneux and Grinvald ${ }^{68}$ explored how the internal dynamics would be modified "after" a brief sensory input. In the barrel cortex of anesthetized rats, with a preparation that displays the stereotypical up and down states, the authors observed that even after a brief single whisker stimulation, this rhythm was significantly perturbed for several seconds, with up events failing to occur, in particular, in the stimulated barrelrelated column [Fig. 4(a)]. As a result, the interaction between internal (recurrent, top-down) and feedforward activities did not appear any more as the smooth integration of two complementary activities, but on the contrary as a competition between orthogonal activities. The authors indeed suggested that "at the onset of a sensory input, some internal messages are silenced to prevent overloading of the processing of relevant incoming sensory information." In addition, this switch in the internal dynamics was also characterized by a transient burst of activity at around $15 \mathrm{~Hz}$ (identified as a thalamo-cortical oscillation) ${ }^{70,71}$ and a transient activity increase of a small fraction of the neurons [both visible in Fig. 4(a); the $\sim 15-\mathrm{Hz}$ activity is marked with gray arrows]. These two patterns occur identically as well after the onset of a longer, sustained stimulation (not visible 


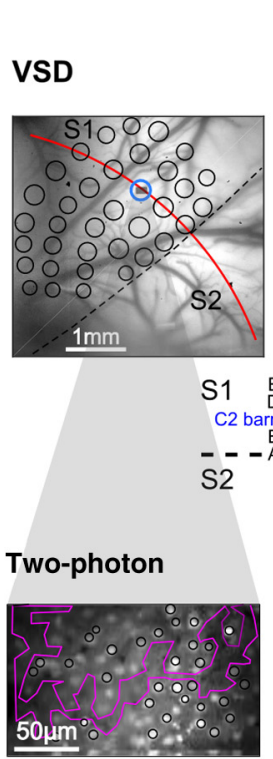

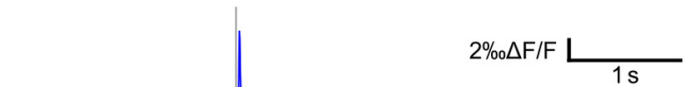
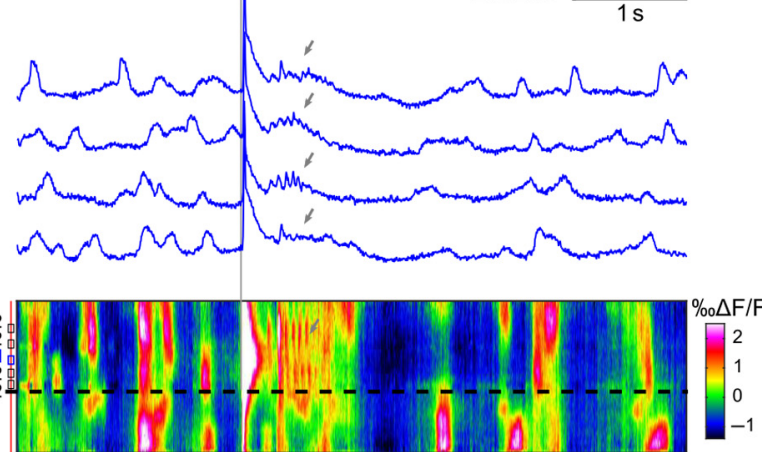

$$
\begin{aligned}
& 49 \text { trials } \times 3 \text { neurons }
\end{aligned}
$$

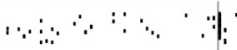

$$
\begin{aligned}
& \therefore \therefore ; \quad, \therefore \text {, }
\end{aligned}
$$

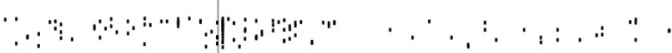

(a)
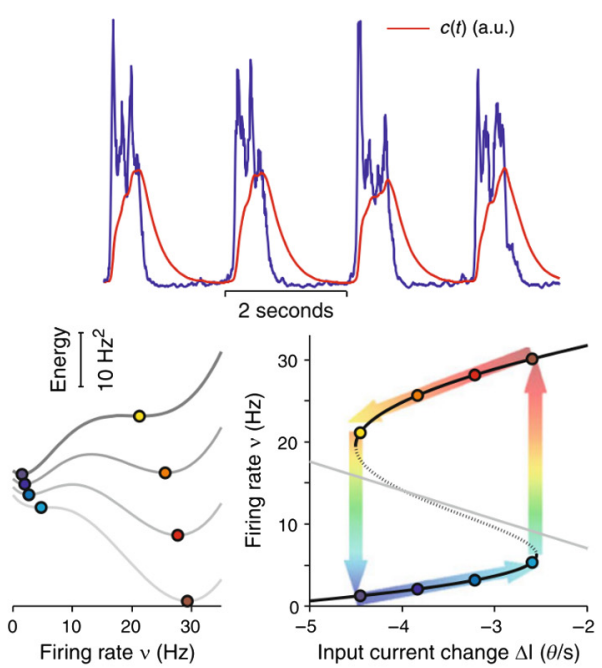

(b)

Fig. 4 Brain dynamics after a sensory input: (a) spontaneous dynamics, response evoked by a brief somatosensory stimulation and poststimulation dynamics, recorded in anesthetized rat barrel cortex using both VSD imaging of population activity and two-photon microscopy of individual neurons spiking activity. VSD signals display four example trials and are extracted from the stimulated barrel location (blue circle in the barrel map on the left); in addition, a spatiotemporal display allows visualizing activity throughout a line (marked in red in the barrel map) crossing both the primary and secondary somatosensory cortices ( 1 1 and S2). Raster plot of spikes extracted from the two-photon recordings displays 49 trials for three different neurons. The main effect of the sensory input on the subsequent dynamics is a decrease or even interruption of the up states appearances for a few seconds (see text for more details). Adapted from Ref. 68 with permission. (b) A model of up and down states generation that involves a fatigue mechanism. (top) Example multiunit activity trace from experiment (blue) and reconstructed fatigue variable $c(t)$. (bottom left): energy landscapes for different levels of activity-dependent adaptation/fatigue; when fatigue is high (top trace), the minimum energy point is an absence of activity. (bottom right): stable (solid branches) and unstable (dotted branch) asymptotic states of firing rate at different fatigue levels here represented as effective changes in the input current to the neurons in the network; colored arrows and circles depict the orbit in the phase plane followed by the network under the relaxation oscillator regime. Adapted from Ref. 69 with permission.

in the figure, see Ref. 68 for details) and thus appear as a stereotyped sequence of events that take place in the presence of a new sensory stream. Finally, the internal activity decrease showed some spatial organization as it was maximal in the stimulated barrel location [visible in Fig. 4(a)], indicating that local mechanisms might be at work.

In this report, emphasis is put on a rupture between pre- and poststimulus onset activities, with the notion that specific switching occurs, affecting subsequent dynamics at the temporal scale of seconds. To this respect, the authors of this review also present in this issue of Neurophotonics a research article of particular interest as it reproduces in an awake monkey V4 area the variability "quenching" reported by Churchland et al. ${ }^{58}$ (yet adding the precision that the activity that is suppressed is a specific global and low-frequency fluctuation). It further shows that this suppression already occurs with maximal strength from the lowest contrast, suggesting that a specific switch occurs rather than a continuous integration.

The notion that an input may cause a switch in the dynamics of a network activity is already present in computational models of the brain ${ }^{65}$ and has been reported in in-vitro studies ${ }^{72-74}$ where microstimulations remarkably induced transitions between up and down, or between synchronized and desynchronized states, as well as in vivo, using nonphysiological stimuli. ${ }^{75}$ Also, it has been known for a long time in the human neuroscience community that sensory inputs alter synchronized rhythms, with the most famous effect being the decrease in alpha rhythms, ${ }^{76-78}$ and that resting-state activity in the DMN decreases upon stimulation. $^{39,42,79}$

It is in fact expected that a new sensory input might cause major changes in the global brain state, switching it, for example, from quiet to active or from asleep to conscious. Studying the details and mechanisms of these switches in addition to the mechanisms of specific rhythms taken in isolation will probably provide new insights on these complex properties of the brain network. As an example, checking how existing models of generation of the up and down fluctuations ${ }^{69,80,81}$ [Fig. 4(b)] would predict not only the evoked responses ${ }^{18,82}$ but also subsequent internal activity changes is warranted.

\subsection{Global Network Changes}

The changes in cortical dynamics induced by the presence of a sensory input occur also at the scale of the full brain, indicative of a change of the subject global state. This is the topic of functional connectivity studies in humans, for which the imaging techniques (EEG, MEG, fMRI, PET), characterized by coarse spatial resolution but access to the whole brain at once, are 
particularly adapted. A set of structurally and functionally connected brain regions specifically deactivated during tasks that demand attention to external stimuli and innovative events has been collectively named the "DMN." Although the understanding of its role in brain function still remains largely elusive, the implication of the DMN in internal modes of cognition (autobiographical memory, self-referential thought, and mindwandering) as well as its alterations in neuropsychiatric disorders is the subject of intense research efforts. ${ }^{42,79}$ The ongoing DMN activity has been reported to be negatively correlated with stimulus-induced responses and perception in humans,${ }^{83,84}$ however, positive correlation has been also observed, ${ }^{85}$ suggesting that the experimental context and the behavioral paradigm strongly impact the link between DMN activity and sensory processing.

\section{Not One but Many Spontaneous Activities}

Altogether, it appears that, despite considerable efforts aimed at studying the spontaneous activity, its functional role remains elusive and might range from low-level maintenance and consolidation of the network ${ }^{86,87}$ to high-level signature of consciousness. ${ }^{39,42,79}$ In particular, even though it is undisputable that ongoing states interfere with sensory processing and are reshaped by learning, direct experimental testing of whether they take an active role in sensory processing remains difficult.

Obviously, it is a pitfall anyway to consider spontaneous activity as a homogeneous phenomenon, as it entails all neural processes, unconscious and conscious, that are not directly (or at least not easily) accessible to probing by identified stimulations or tasks. Even the apparent same rhythms in different contexts can in fact display important structural differences, as was shown with slow frequency activity that appeared to be more local during slow wave sleep compared to during quiet wakefulness. ${ }^{88,89}$

However, the impressive development of in vivo optical methods, pioneered in particular by Grinvald, which allow probing cortical spatiotemporal dynamics at the single-trial level in both anesthetized and awake preparations, will undoubtedly keep on bringing precious keys to further unravel the functional interplay between internal dynamics and sensory inputs in cortical networks.

\section{Disclosure}

No conflicts of interest, financial or otherwise, are declared by the authors.

\section{Acknowledgments}

The authors are funded by the Centre National de la Recherche Scientifique, France.

\section{References}

1. A. Arieli et al., "Coherent spatiotemporal patterns of ongoing activity revealed by real-time optical imaging coupled with single-unit recording in the cat visual cortex," J. Neurophysiol. 73(5), 2072-2093 (1995).

2. A. Arieli et al., "Dynamics of ongoing activity: explanation of the large variability in evoked cortical responses," Science 273(5283), 1868-1871 (1996).

3. G. Hesselmann et al., "Spontaneous local variations in ongoing neural activity bias perceptual decisions," Proc. Natl. Acad. Sci. U. S. A. 105(31), 10984-10989 (2008).

4. C. Bennett, S. Arroyo, and S. Hestrin, "Subthreshold mechanisms underlying state-dependent modulation of visual responses," Neuron 80(2), 350-357 (2013).
5. M. L. Schölvinck et al., "Cortical state determines global variability and correlations in visual cortex," J. Neurosci. 35(1), 170-178 (2015).

6. S. Crochet and C. C. H. Petersen, "Correlating whisker behavior with membrane potential in barrel cortex of awake mice," Nat. Neurosci. 9(5), 608-610 (2006).

7. C. C. H. Petersen et al., "Interaction of sensory responses with spontaneous depolarization in layer $2 / 3$ barrel cortex," Proc. Natl. Acad. Sci. U. S. A. 100(23), 13638-13643 (2003).

8. E. F. Civillico and D. Contreras, "Spatiotemporal properties of sensory responses in vivo are strongly dependent on network context," Front. Syst. Neurosci. 6, 25 (2012).

9. M. Zhou et al., "Scaling down of balanced excitation and inhibition by active behavioral states in auditory cortex," Nat. Neurosci. 17(6), 841-850 (2014).

10. A. Hasenstaub, R. N. S. Sachdev, and D. A. McCormick, "State changes rapidly modulate cortical neuronal responsiveness," J. Neurosci. 27(36), 9607-9622 (2007).

11. S. A. Romano et al., "Spontaneous neuronal network dynamics reveal circuit's functional adaptations for behavior," Neuron 85(5), 1070-1085 (2015).

12. B. Haider et al., "Enhancement of visual responsiveness by spontaneous local network activity in vivo," J. Neurophysiol. 97(6), 4186-4202 (2007).

13. K. D. Harris and A. Thiele, "Cortical state and attention," Nat. Rev. Neurosci. 12(9), 509-523 (2011).

14. I. Ferezou, S. Bolea, and C. C. H. Petersen, "Visualizing the cortical representation of whisker touch: voltage-sensitive dye imaging in freely moving mice," Neuron 50(4), 617-629 (2006).

15. R. N. S. Sachdev, "Effect of subthreshold up and down states on the whisker-evoked response in somatosensory cortex," J. Neurophysiol. 92(6), 3511-3521 (2004).

16. I. Nauhaus et al., "Stimulus contrast modulates functional connectivity in visual cortex," Nat. Neurosci. 12(1), 70-76 (2008).

17. T. Altwegg-Boussac et al., "Excitability and responsiveness of rat barrel cortex neurons in the presence and absence of spontaneous synaptic activity in vivo," J. Physiol. 592(16), 3577-3595 (2014).

18. R. Reig et al., "Gain modulation of synaptic inputs by network state in auditory cortex in vivo," J. Neurosci. 35(6), 2689-2702 (2015).

19. M. Pachitariu et al., "State-dependent population coding in primary auditory cortex," J. Neurosci. 35(5), 2058-2073 (2015).

20. I. Timofeev, D. Contreras, and M. Steriade, "Synaptic responsiveness of cortical and thalamic neurones during various phases of slow sleep oscillation in cat," J. Physiol. 494(Pt. 1), 265-278 (1996).

21. R. Azouz and C. M. Gray, "Cellular mechanisms contributing to response variability of cortical neurons in vivo," J. Neurosci. 19(6), 2209-2223 (1999).

22. P.-O. Polack, J. Friedman, and P. Golshani, "Cellular mechanisms of brain state-dependent gain modulation in visual cortex," Nat. Neurosci. 16(9), 1331-1339 (2013).

23. M. R. Deweese and A. M. Zador, "Shared and private variability in the auditory cortex," J. Neurophysiol. 92(3), 1840-1855 (2004).

24. M. Steriade, D. McCormick, and T. Sejnowski, "Thalamocortical oscillations in the sleeping and aroused brain," Science 262(5134), 679-685 (1993).

25. E. E. Fanselow and M. A. Nicolelis, "Behavioral modulation of tactile responses in the rat somatosensory system," J. Neurosci. 19(17), 76037616 (1999).

26. H. Hentschke, F. Haiss, and C. Schwarz, "Central signals rapidly switch tactile processing in rat barrel cortex during whisker movements," Cereb. Cortex 16(8), 1142-1156 (2005).

27. G. H. Otazu et al., "Engaging in an auditory task suppresses responses in auditory cortex," Nat. Neurosci. 12(5), 646-654 (2009).

28. D. M. Schneider, A. Nelson, and R. Mooney, "A synaptic and circuit basis for corollary discharge in the auditory cortex," Nature $\mathbf{5 1 3}$ (7517), 189-194 (2014).

29. A. V. Egorov and A. Draguhn, "Development of coherent neuronal activity patterns in mammalian cortical networks: common principles and local hetereogeneity," Mech. Dev. 130(6-8), 412-423 (2013).

30. M. L. Andermann et al., "Functional specialization of mouse higher visual cortical areas," Neuron 72(6), 1025-1039 (2011).

31. Y. Fu et al., "A cortical circuit for gain control by behavioral state," Cell 156(6), 1139-1152 (2014). 
32. C. M. Niell and M. P. Stryker, "Modulation of visual responses by behavioral state in mouse visual cortex," Neuron 65(4), 472-479 (2010).

33. G. B. Keller, T. Bonhoeffer, and M. Hübener, "Sensorimotor mismatch signals in primary visual cortex of the behaving mouse," Neuron 74(5), 809-815 (2012).

34. J. Reimer et al., "Pupil fluctuations track fast switching of cortical states during quiet wakefulness," Neuron 84(2), 355-362 (2014).

35. A. M. Lee et al., "Identification of a brainstem circuit regulating visual cortical state in parallel with locomotion," Neuron 83(2), 455-466 (2014).

36. S. Sachidhanandam et al., "Membrane potential correlates of sensory perception in mouse barrel cortex," Nat. Neurosci. 16(11), 1671-1677 (2013).

37. M. D. Fox et al., "Coherent spontaneous activity accounts for trial-totrial variability in human evoked brain responses," Nat. Neurosci. 9(1), 23-25 (2006).

38. G. Northoff, P. Qin, and T. Nakao, "Rest-stimulus interaction in the brain: a review," Trends Neurosci. 33(6), 277-284 (2010).

39. M. E. Raichle, "Two views of brain function," Trends Cognit. Sci. 14(4), 180-190 (2010).

40. S. Dehaene and J.-P. Changeux, "Experimental and theoretical approaches to conscious processing," Neuron 70(2), 200-227 (2011).

41. S. Dehaene and J.-P. Changeux, "Ongoing spontaneous activity controls access to consciousness: a neuronal model for inattentional blindness," PLoS Biol. 3(5), e141 (2005).

42. M. E. Raichle, "The restless brain: how intrinsic activity organizes brain function," Philos. Trans. R. Soc. London B, Biol. Sci. 370(1668), 20140172 (2015).

43. M. Tsodyks et al., "Linking spontaneous activity of single cortical neurons and the underlying functional architecture," Science 286(5446), 1943-1946 (1999).

44. T. Kenet et al., "Spontaneously emerging cortical representations of visual attributes," Nature 425(6961), 954-956 (2003).

45. F. Han, N. Caporale, and Y. Dan, "Reverberation of recent visual experience in spontaneous cortical waves," Neuron 60(2), 321-327 (2008).

46. P. Berkes et al., "Spontaneous cortical activity reveals hallmarks of an optimal internal model of the environment," Science 331(6013), 83-87 (2011).

47. M. H. Mohajerani et al., "Spontaneous cortical activity alternates between motifs defined by regional axonal projections," Nat. Neurosci. 16(10), 1426-1435 (2013).

48. S. Xu et al., "Activity recall in a visual cortical ensemble," Nat. Neurosci. 15(3), 449-455 (2012).

49. D. R. Euston, M. Tatsuno, and B. L. McNaughton, "Fast-forward playback of recent memory sequences in prefrontal cortex during sleep," Science 318(5853), 1147-1150 (2007).

50. E. J. B. Contreras et al., "Formation and reverberation of sequential neural activity patterns evoked by sensory stimulation are enhanced during cortical desynchronization," Neuron 79(3), 555-566 (2013).

51. D. Ji and M. A. Wilson, "Coordinated memory replay in the visual cortex and hippocampus during sleep," Nat. Neurosci. 10(1), 100-107 (2007).

52. M. A. Wilson and B. L. McNaughton, "Reactivation of hippocampal ensemble memories during sleep," Science 265(5172), 676-679 (1994).

53. W. E. Skaggs and B. L. McNaughton, "Replay of neuronal firing sequences in rat hippocampus during sleep following spatial experience," Science 271(5257), 1870-1873 (1996).

54. G. R. Sutherland and B. McNaughton, "Memory trace reactivation in hippocampal and neocortical neuronal ensembles," Curr. Opin. Neurobiol. 10(2), 180-186 (2001).

55. A. G. Blankenship and M. B. Feller, "Mechanisms underlying spontaneous patterned activity in developing neural circuits," Nat. Rev. Neurosci. 11(1), 18-29 (2009).

56. H. J. Luhmann, "Review of imaging network activities in developing rodent cerebral cortex in vivo," Neurophotonics 4(3), 031202 (2016).

57. C. M. Lewis et al., "Learning sculpts the spontaneous activity of the resting human brain," Proc. Natl. Acad. Sci. U. S. A. 106(41), 17558-17563 (2009).

58. M. M. Churchland et al., "Stimulus onset quenches neural variability: a widespread cortical phenomenon," Nat. Neurosci. 13(3), 369-378 (2010).
59. J. Fiser et al., "Statistically optimal perception and learning: from behavior to neural representations," Trends Cognit. Sci. 14(3), 119-130 (2010).

60. L. Buesing et al., "Neural dynamics as sampling: a model for stochastic computation in recurrent networks of spiking neurons," PLoS Comput. Biol. 7(11), e1002211 (2011).

61. L. J. Borg-Graham, C. Monier, and Y. Fregnac, "Visual input evokes transient and strong shunting inhibition in visual cortical neurons," Nature 393(6683), 369-373 (1998).

62. K. Rajan, L. F. Abbott, and H. Sompolinsky, "Stimulus-dependent suppression of chaos in recurrent neural networks," Phys. Rev. E 82(Pt. 1), 011903 (2010).

63. L. F. Abbott, K. Rajan, and H. Sompolinsky, "Interactions between intrinsic and stimulus-dependent activity in recurrent neural networks," in The Dynamic Brain: An Exploration of Neuronal Variability and Its Functional Significance, M. Ding and D. Glanzman, Eds., pp. 65-82, Oxford University Press, New York (2011).

64. B. White, L. F. Abbott, and J. Fiser, "Suppression of cortical neural variability is stimulus- and state-dependent," J. Neurophysiol. 108(9), 2383-2392 (2012).

65. G. Deco and V. K. Jirsa, "Ongoing cortical activity at rest: criticality, multistability, and ghost attractors," J. Neurosci. 32(10), 3366-3375 (2012).

66. A. Ponce-Alvarez et al., "Task-driven activity reduces the cortical activity space of the brain: experiment and whole-brain modeling," PLoS Comput. Biol. 11(8), e1004445 (2015).

67. D. L. Ringach, "Spontaneous and driven cortical activity: implications for computation," Curr. Opin. Neurobiol. 19(4), 439-444 (2009).

68. T. Deneux and A. Grinvald, "Milliseconds of sensory input abruptly modulate the dynamics of cortical states for seconds," Cereb. Cortex 1-15 (2016).

69. M. Mattia, "Exploring the spectrum of dynamical regimes and timescales in spontaneous cortical activity," Cognit. Neurodyn. 6(3), 239250 (2012).

70. D. Derdikman et al., "Imaging spatiotemporal dynamics of surround inhibition in the barrels somatosensory cortex," J. Neurosci. 23(8), 3100-3105 (2003).

71. M. M. Halassa et al., "Selective optical drive of thalamic reticular nucleus generates thalamic bursts and cortical spindles," Nat. Neurosci. 14(9), 1118-1120 (2011).

72. Y. Shu, A. Hasenstaub, and D. A. McCormick, "Turning on and off recurrent balanced cortical activity," Nature 423(6937), 288-293 (2003).

73. J. N. MacLean et al., "Internal dynamics determine the cortical response to thalamic stimulation," Neuron 48(5), 811-823 (2005).

74. S. Fujisawa, N. Matsuki, and Y. Ikegaya, "Single neurons can induce phase transitions of cortical recurrent networks with multiple internal States," Cereb. Cortex 16(5), 639-654 (2006).

75. F. Kasanetz, L. A. Riquelme, and M. G. Murer, "Disruption of the twostate membrane potential of striatal neurones during cortical desynchronisation in anaesthetised rats," J. Physiol. 543(Pt. 2), 577-589 (2002).

76. E. Callaway and R. S. Layne, "Interaction between the visual evoked response and two spontaneous biological rhythms: the EEG alpha cycle and the cardiac arousal cycle," Ann. N. Y. Acad. Sci. 112, 421-431 (1964).

77. G. Pfurtscheller and W. Klimesch, "Functional topography during a visuoverbal judgment task studied with event-related desynchronization mapping," J. Clin. Neurophysiol. 9(1), 120-131 (1992).

78. M. Siegel, T. H. Donner, and A. K. Engel, "Spectral fingerprints of large-scale neuronal interactions," Nat. Rev. Neurosci. 13(2), 121-134 (2012).

79. R. L. Buckner, J. R. Andrews-Hanna, and D. L. Schacter, "The brain's default network: anatomy, function, and relevance to disease," Ann. N. Y. Acad. Sci. 1124(1), 1-38 (2008).

80. K. E. Poskanzer and R. Yuste, "Astrocytes regulate cortical state switching in vivo," Proc. Natl. Acad. Sci. U. S. A. 113(19), E2675-E2684 (2016).

81. R. Yuste et al., "The cortex as a central pattern generator," Nat. Rev. Neurosci. 6(6), 477-483 (2005).

82. C. Curto et al., "A simple model of cortical dynamics explains variability and state dependence of sensory responses in urethane-anesthetized auditory cortex," J. Neurosci. 29(34), 10600-10612 (2009). 
83. M. D. Greicius and V. Menon, "Default-mode activity during a passive sensory task: uncoupled from deactivation but impacting activation," J. Cognit. Neurosci. 16(9), 1484-1492 (2004).

84. M. Boly et al., "Baseline brain activity fluctuations predict somatosensory perception in humans," Proc. Natl. Acad. Sci. U. S. A. 104(29), 12187-12192 (2007).

85. S. Sadaghiani, G. Hesselmann, and A. Kleinschmidt, "Distributed and antagonistic contributions of ongoing activity fluctuations to auditory stimulus detection," J. Neurosci. 29(42), 13410-13417 (2009).

86. L. Marshall et al., "Boosting slow oscillations during sleep potentiates memory," Nature 444(7119), 610-613 (2006).

87. M. V. Sanchez-Vives and M. Mattia, "Slow wave activity as the default mode of the cerebral cortex," Arch. Ital. Biol. 152(2-3), 147-155 (2015).

88. U. Olcese et al., "Spike-based functional connectivity in cerebral cortex and hippocampus: loss of global connectivity is coupled to preservation of local connectivity during non-REM sleep," J. Neurosci. 36(29), 7676-7692 (2016).

89. L. M. J. Fernandez et al., "Highly dynamic spatiotemporal organization of low-frequency activities during behavioral states in the mouse cerebral cortex," Cereb. Cortex 1-19 (2016).
Isabelle Ferezou studies the processing of tactile sensory information in the mouse cortex using voltage-sensitive dye (VSD) imaging in Daniel Shulz Lab, Unité de Neurosciences Information et Complexité (UNIC), Centre National de la Recherche Scientifique (CNRS), France. During her PhD supervised by Bertrand Lambolez at ESPCI, Paris, France, she studied GABAergic interneurons using single-cell RT-PCR after patch-clamp in vitro. She was then trained in in vivo VSD imaging and electrophysiology in the laboratory of Professor Carl Petersen at EPFL, Switzerland.

Thomas Deneux develops data analysis methods for high-throughput recordings of neural activity at UNIC, CNRS, France. His PhD supervised by Olivier Faugeras at the Institut National de Recherche en Informatique et Automatique, Paris, France, focused on human brain data analysis, whereas he studied spontaneous activity and multisensory integration using VSD and two-photon microscopy during his postdoctoral trainings supervised by Amiram Grinvald at the Weizmann Institute, Rehovot, Israel, Ivo Vanzetta at Institut de Neurosciences de la Timone, Marseille, France, and Brice Bathellier at CNRS-UNIC, Paris, France. 